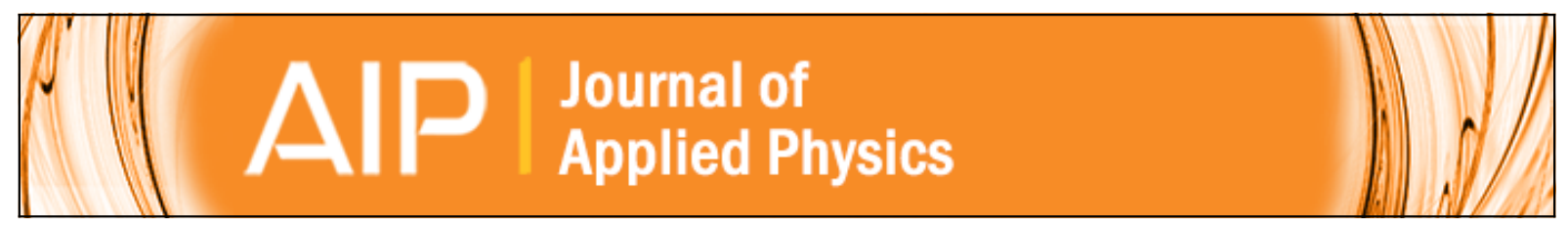

\title{
Competition of exchange and crystal field interactions in cerium monopnictides and monochalcogenides
}

Eric M. Collins, Nicholas Kioussis, Say Peng Lim, and Bernard R. Cooper

Citation: Journal of Applied Physics 87, 5143 (2000); doi: 10.1063/1.373276

View online: http://dx.doi.org/10.1063/1.373276

View Table of Contents: http://scitation.aip.org/content/aip/journal/jap/87/9?ver=pdfcov

Published by the AIP Publishing

\section{Articles you may be interested in}

Heavy fermion Ce3Co4Sn13 compound under pressure

J. Appl. Phys. 117, 17E307 (2015); 10.1063/1.4913304

First-principles study of electronic structure and magnetic properties of $\mathrm{Cu}$-doped $\mathrm{CeO} 2$

J. Appl. Phys. 112, 083702 (2012); 10.1063/1.4759359

Magnetic-field-induced effects in the electronic structure of itinerant $d$ - and $\mathrm{f}$-metal systems

Low Temp. Phys. 35, 638 (2009); 10.1063/1.3224723

Crystal field effects in Ce Os $4 \mathrm{Sb} 12$ as determined by inelastic neutron scattering

Appl. Phys. Lett. 89, 082508 (2006); 10.1063/1.2264336

Enlarged paramagnetism by electron-electron exchange interactions in n-type modulation doped $\mathrm{Cd} 1-\mathrm{x} \mathrm{Mn} \mathrm{x}$ $\mathrm{Te} / \mathrm{Cd} 1-\mathrm{x}-\mathrm{y} \mathrm{Mn} \mathrm{x} \mathrm{Mg}$ y Te single quantum wells

J. Appl. Phys. 85, 5947 (1999); 10.1063/1.370000

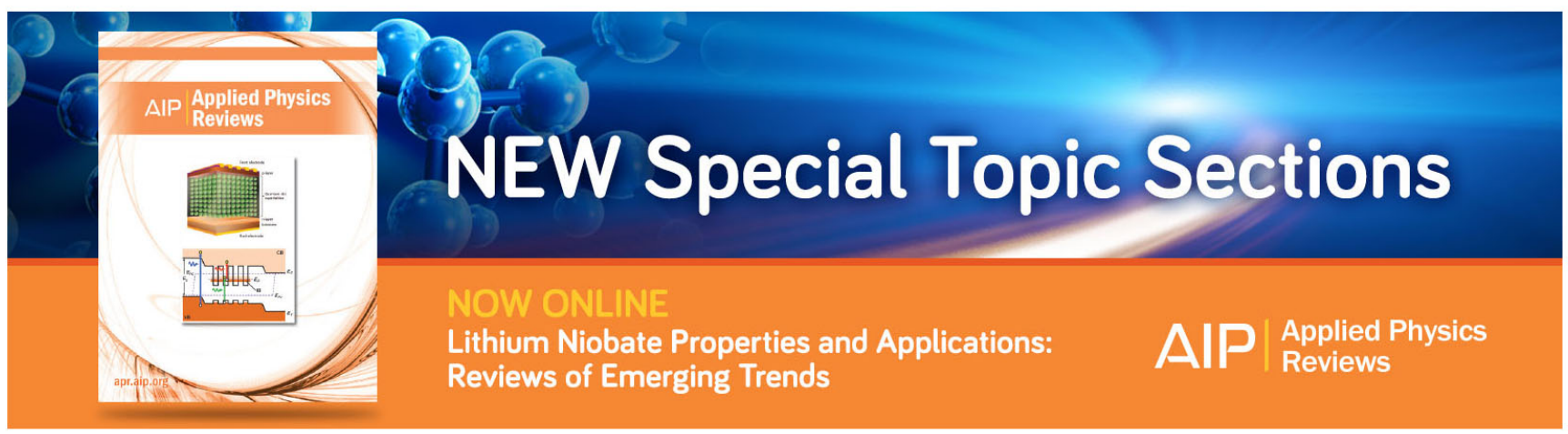




\title{
Competition of exchange and crystal field interactions in cerium monopnictides and monochalcogenides
}

\author{
Eric M. Collins, Nicholas Kioussis, ${ }^{\text {a) }}$ and Say Peng Lim \\ Department of Physics and Astronomy, California State University Northridge, Northridge, \\ California 91330-8268 \\ Bernard R. Cooper \\ Department of Physics, West Virginia University, Morgantown, West Virginia 26506
}

\begin{abstract}
We have applied two ab initio based methods to study the dramatic change of magnetic properties across a series of strongly correlated electron systems consisting of cerium monopnictides and monochalcogenides. While, the fully ab initio method gives good results for the magnetic moments in the lighter chalcogenides, it entirely fails to give, even qualitatively, the trend of their unusual magnetic behavior. On the other hand, the second approach, which explicitly takes into account the interplay between the hybridization, the Coulomb exchange, and the crystal-field interactions, gives results in excellent agreement with experiment for all compounds in the series, including the moment collapse from $\mathrm{CeSb}$ to $\mathrm{CeTe}$ and the trend of moments and ordering temperatures across the series. () 2000 American Institute of Physics. [S0021-8979(00)65008-9]
\end{abstract}

The isostructural (rock-salt structure) series of the cerium monopnictides $\mathrm{Ce} X(X=\mathrm{P}, \mathrm{As}, \mathrm{Sb}, \mathrm{Bi})$ and monochalcogenides $(X=\mathrm{S}, \mathrm{Se}, \mathrm{Te})$ have become prototype model systems for study, because of their unusual magnetic properties. This series of strongly correlated electron systems offers the opportunity to vary systematically, through chemical pressure, the lattice constant and the cerium separation on going down the pnictogen or chalcogen column, and hence tailor the degree of $4 f$ localization from the strongly localized limit in the heavier systems to to the weakly localized limit in the lighter systems. ${ }^{1-7}$ The sensitivity of the hybridization, Coulomb exchange, and crystal-field (CF) interactions to the chemical environment gives rise to a variety of unusual magnetic properties.

This class of systems exhibits strong magnetic anisotropy which changes from the $\langle 001\rangle$ direction in the pnictides to the $\langle 111\rangle$ direction in the chalcogenides. The low temperature magnetic moment increases with increasing lattice constant for the pnictides from $0.80 \mu_{B}$ in $\mathrm{CeP}$ to $2.1 \mu_{B}$ in $\mathrm{CeBi},{ }^{1-2}$ and decreases with increasing lattice constant for the chalcogenides from $0.57 \mu_{B}$ in $\mathrm{CeS}$ to $0.3 \mu_{B}$ in CeTe. ${ }^{3-5}$ The magnetic moment collapse from $\mathrm{CeSb}$ to $\mathrm{CeTe}$, with both systems having about the same lattice constant, is indicative of the sensitivity of the exchange interactions to the chemical environment. The ordering temperature increases from $8 \mathrm{~K}$ in $\mathrm{CeP}$ to $26 \mathrm{~K}$ in $\mathrm{CeBi}$ for the pnictides, whereas it decreases from $8.4 \mathrm{~K}$ in $\mathrm{CeS}$ to an unusually low $2.2 \mathrm{~K}$ in CeTe. $^{1-5}$

An unusual feature of the cerium series is the large suppression of the CF splitting of the $\mathrm{Ce}^{3+}$ free-ion $4 f_{5 / 2}$ multiplet from values expected from the behavior of the heavier isostructural rare-earth monopnictides. ${ }^{8}$ In both the cerium monopnictides and monochalcogenides, the CF splitting between the $\Gamma_{7}$ doublet and the $\Gamma_{8}$ quartet decreases with in-

\footnotetext{
a) Author to whom correspondence should be addressed; electronic mail: nkioussi@newton.csun.edu
}

creasing anion size, from $150 \mathrm{~K}$ for $\mathrm{CeP}$ to $8 \mathrm{~K}$ in $\mathrm{CeBi}$ and from $140 \mathrm{~K}$ for $\mathrm{CeS}$ to $32 \mathrm{~K}$ for CeTe, and it is about the same in both series, a rather surprising result in view of the additional valence electron on the chalcogen ion. ${ }^{9}$ Neutron scattering experiments have shown ${ }^{10}$ that the $\Gamma_{7}$ doublet is the $\mathrm{CF}$ ground state in all the cerium monopnictides and monochalcogenides.

The purpose of this work is to understand the underlying mechanisms responsible for the unusual magnetism in this class of systems and to investigate the interplay of exchange and crystal field interactions. We have employed two $a b$ initio based approaches to calculate the magnetic properties of these compounds. First, we have carried out ab initio spin polarized electronic structure calculations based on the full potential linear muffin tin orbital (FPLMTO) method ${ }^{11}$ using (1) only the spin polarization, with the orbital polarization included only through spin-orbit coupling, and (2) both the spin and orbital polarization correction. ${ }^{12}$ Second, we apply an $a b$ initio based approach synthesizing (1) a phenomenological theory of orbitally driven magnetism based on the Anderson and Kondo lattice model which incorporates explicitly the hybridization induced, the Coulomb exchange, and the crystal field interactions on an equal footing, and (2) FPLMTO electronic structure calculations allowing a first principles evaluation of all the parameters entering the model Hamiltonian, except for the crystal field interaction. To date, our attempts ${ }^{13}$ to determine the CF splitting for this class of systems on a fully $a b$ initio basis have not proven successful, and thus we use the experimental ${ }^{10} \mathrm{CF}$ value for each compound. In contrast to the first method, in the FPLMTO evaluation of the parameters entering the model Hamiltonian, the $4 f$ states are treated as localized, atomic-like, states and are placed in the core not being allowed to hybridize with the non- $f$ states, in order to be consistent with the treatment of the $f$ states in the Anderson-Kondo Hamiltonian. ${ }^{14-16}$ It should be emphasized that in the second method the band energies, the $f$-state energies, the on-site Coulomb repulsion 
$U$, the hybridization matrix elements, $V_{k m}$, and the band- $f$ Coulomb exchange $\quad J_{m m^{\prime}}\left(\mathbf{k}, \mathbf{k}^{\prime}\right)=\left\langle\phi_{\kappa}^{*}\left(r_{1}\right) \psi_{m}^{*}\left(r_{2}\right)\right| 1 /$ $r_{12}\left|\psi_{m^{\prime}}\left(r_{1}\right) \phi_{\kappa^{\prime}}\left(r_{2}\right)\right\rangle$ are evaluated from nonspin polarized FPLMTO calculations. Comparison of the two methods tests the limitations of these $a b$ initio based calculations as the degree of $4 f$ localization is varied across the series, and reveals the failure of standard $a b$ initio electronic structure calculations based on density functional theory within the local density approximation to predict, even qualitatively, the trend of magnetic properties in these strongly correlated electron systems. In the past, we have applied the second method to investigate the effect of hybridization-induced exchange interactions ${ }^{14,15}$ and the effect of both the hybridization and Coulomb exchange interactions ${ }^{16}$ on the magnetic properties of the heavier cerium pnictides and chalcogenides $(\mathrm{CeBi}$, $\mathrm{CeSb}$, and $\mathrm{CeTe}$ ). However, these calculations did not take into account the crystal field interaction and employed a warped muffin-tin LMTO calculation for the parameters entering the model. The very good agreement found ${ }^{16}$ with experiment for the low-temperature magnetic moment and ordering temperature is due to the fact that the $\mathrm{CF}$ interaction in the heavier systems is smaller (about $8 \mathrm{~K}$ ) than the exchange interactions. We have generalized our $a b$ initio based method to include the exchange (both hybridization and Coulomb exchange) and crystal field interactions on an equal footing and to employ a full potential LMTO evaluation of the exchange parameters. While the effect of the full potential on both the hybridization and Coulomb exchange interactions is small, the inclusion of the CF interaction will be shown to play a role as important as the exchange interactions for understanding and predicting the unusual magnetic properties, as one tunes the degree of $4 f$ localization across this series of strongly correlated electron systems. Because the size of both the hybridization and Coulomb exchange matrix elements are much smaller (about $2 \mathrm{meV}$ ) than the intra-atomic cerium Coulomb interaction $U(6 \mathrm{eV})$, one can apply the perturbation theory and evaluate the anisotropic two-ion exchange $6 \times 6$ matrices. ${ }^{14-16}$ The exchange interactions have three contributions: the pure Coulomb exchange interaction term proportional to $J_{m m^{\prime}}^{2}\left(\mathbf{k}, \mathbf{k}^{\prime}\right)$, the hybridization-mediated exchange interaction term proportional to $V_{k m}^{4}$, and the cross term proportional to $V_{k m}^{2} J_{m m^{\prime}}\left(\mathbf{k}, \mathbf{k}^{\prime}\right)$.

We next discuss the necessity of including the CF interaction term in the phenomenological Hamiltonian, so we can investigate the interplay of exchange and CF interactions on an equal footing as we chemically tune the various pertinent interactions across the series. In the second approach, the $4 f$ states are treated as core states, and as such, they interact only with the spherical component of the effective oneelectron potential. Thus, the interaction of the atomic-like $4 f$ state with the nonspherical components of the potential, giving rise to the CF splitting, is not explicitly included. Thus, the $\mathrm{CF}$ interaction is included in the phenomenological theory through the inclusion of the $\mathrm{CF}$ interaction, $H_{\mathrm{CF}}$ $=B_{4}\left(O_{4}^{0}+5 O_{4}^{4}\right)$ where the $O_{4}^{0}$ and $O_{4}^{4}$ are Stevens equivalence operators. ${ }^{17}$ (A positive $B_{4}$ value gives the $\Gamma_{7}$ ground state, experimentally observed.) In the absence of an $a b$ initio value of the $\mathrm{CF}$ interaction in this class of strongly cor-
TABLE I. Values of the calculated and experimental (Refs. 1-4) magnetic moments for the cerium chalcogenides and pnictides in units of $\mu_{B}$. Listed are the LMTO values for the spin moment $\mu_{S}$, the orbital moment $\mu_{L}$, and total moment $\mu$, for the spin polarized only calculation and for the calculation with spin polarization and orbital polarization correction.

\begin{tabular}{|c|c|c|c|c|c|c|c|}
\hline & \multicolumn{3}{|c|}{$\mathrm{FP}+\mathrm{SP}$} & \multicolumn{3}{|c|}{$\mathrm{FP}+\mathrm{SP}+\mathrm{OP}$} & \multirow{2}{*}{$\begin{array}{c}\text { EXPT } \\
\mu\end{array}$} \\
\hline & $\mu_{S}$ & $\mu_{L}$ & $\mu$ & $\mu_{S}$ & $\mu_{L}$ & $\mu$ & \\
\hline $\mathrm{CeS}$ & -1.00 & 0.91 & -0.09 & -1.24 & 1.99 & 0.75 & 0.57 \\
\hline $\mathrm{CeSe}$ & -1.08 & 1.02 & -0.06 & -1.26 & 2.07 & 0.81 & 0.57 \\
\hline CeTe & -1.15 & 1.28 & 0.07 & -1.31 & 2.29 & 0.98 & 0.30 \\
\hline $\mathrm{CeP}$ & -0.80 & 0.55 & -0.25 & -0.85 & 1.27 & 0.43 & 0.80 \\
\hline CeAs & -0.84 & 0.64 & -0.20 & -0.85 & 1.42 & 0.57 & 0.80 \\
\hline $\mathrm{CeSb}$ & -0.86 & 0.74 & -0.12 & -0.91 & 1.61 & 0.70 & 2.06 \\
\hline $\mathrm{CeBi}$ & -0.86 & 0.74 & -0.12 & -0.95 & 1.69 & 0.74 & 2.10 \\
\hline
\end{tabular}

related electron systems, ${ }^{13}$ the $\mathrm{CF}$ splitting, $\Delta_{\mathrm{CF}}=360 B_{4}$, is set to the experimental values, listed in Table III. ${ }^{10}$ With the two-ion interactions having been determined, the lowtemperature magnetic moment and the ordering temperature can be determined by means of a mean field calculation.

In Table I we present the calculated values of the zerotemperature cerium magnetic moment from the FPLMTO electronic structure calculations. Listed in the table are values both with and without the orbital polarization correction taken into account. Note, the importance of including the orbital polarization in these $4 f$ correlated electron systems. In all cases, the orbital polarization is found to be opposite to the spin polarization, as expected. Comparison of the total energies gives that the magnetic anisotropy changes from the $\langle 001\rangle$ direction in the pnictides to the $\langle 111\rangle$ in the chalcogenides, in agreement with experiment. On the other hand, except perhaps for the lighter chalcogenides ( $\mathrm{CeS}$ and $\mathrm{CeSe}$ ), comparison of the $a b$ initio and experimental values for the magnetic moment indicates the failure of the local density approximation (LDA) calculations to treat properly the correlation effects of the $4 f$ states (treated as band states) in this series of strongly correlated electron systems. Furthermore, the pure $a b$ initio calculations fail to predict the large moment collapse from $\mathrm{CeSb}$ to $\mathrm{CeTe}$, the latter being described as an incipient heavy Fermion system.

In Table II, listed are values of the $m=m^{\prime}=1 / 2$ matrix elements (characteristic matrix elements of the $6 \times 6$ exchange interaction matrix) for the first three nearest-neighbor shells for the light ( $\mathrm{CeP}$ and $\mathrm{CeS})$ and the heavier compounds $(\mathrm{CeSb}$ and $\mathrm{CeTe})$. Listed separately in this table are the three contributions to the exchange interaction from hybridization $\left(V^{4}\right)$, Coulomb exchange $\left(J^{2}\right)$, and the cross term. It is important to note that while the Coulomb exchange interactions dominate the magnetic behavior for the heavier, more localized, $4 f$ systems, the opposite is true for the lighter, more delocalized, systems where the hybridization exchange interactions dominate the magnetic behavior. This change of behavior of the exchange interactions is a result of the sensitivity of the hybridization and Coulomb exchange on the degree of $4 f$ localization. Equally important, is that while all nearest-neighbor exchange interactions are ferromagnetic for $\mathrm{CeSb}$, there is an interplay between ferromagnetic and antiferromagnetic interactions for $\mathrm{CeTe}$, 
TABLE II. Listed below are characteristic matrix elements of the $6 \times 6$ two-ion interaction matrix $\left[E_{m_{a} m_{a}^{\prime} m_{b}^{\prime}}^{\prime}\left(\mathbf{R}_{b}-\mathbf{R}_{a}\right)\right]$ of the $f$ electrons for a cross section of the cerium compounds. Values of the hybridization induced $\left(E_{V^{4}}\right)$, cross terms $\left(E_{V^{2} J}\right)$, and pure Coulomb exchange $\left(E_{J^{2}}\right)$ interactions are listed for the first, second, and third nearest cerium neighbors in units of $\mathrm{K}$. the listed elements are the $m=m^{\prime}= \pm 1 / 2$.

\begin{tabular}{|c|c|c|c|c|c|c|}
\hline & \multicolumn{3}{|c|}{$\mathrm{CeP}$} & \multicolumn{3}{|c|}{$\mathrm{CeS}$} \\
\hline & $E_{V^{4}}$ & $E_{V^{2} J}$ & $E_{J^{2}}$ & $E_{V^{4}}$ & $E_{V^{2} J}$ & $E_{J^{2}}$ \\
\hline$R=\left(\frac{1}{2} \frac{1}{2} 0\right)$ & 2.23 & 0.64 & 1.53 & 0.85 & -0.40 & 1.50 \\
\hline$R=\left(\begin{array}{lll}1 & 0 & 0\end{array}\right)$ & 6.39 & 0.27 & 1.65 & -1.60 & 0.04 & -0.80 \\
\hline \multirow[t]{3}{*}{$R=\left(1 \frac{1}{2} \frac{1}{2}\right)$} & -0.08 & -0.02 & 0.16 & 0.38 & -0.16 & 0.13 \\
\hline & \multicolumn{3}{|c|}{$\mathrm{CeSb}$} & \multicolumn{3}{|c|}{ CeTe } \\
\hline & $E_{V^{4}}$ & $E_{V^{2} J}$ & $E_{J^{2}}$ & $E_{V^{4}}$ & $E_{V^{2} J}$ & $E_{J^{2}}$ \\
\hline$R=\left(\frac{1}{2} \frac{1}{2} 0\right)$ & 0.70 & 0.34 & 7.30 & 0.17 & -0.19 & 2.90 \\
\hline$R=\left(\begin{array}{lll}1 & 0 & 0\end{array}\right)$ & 2.07 & 0.07 & 10.21 & -0.19 & 0.04 & -1.69 \\
\hline$R=\left(1 \frac{1}{2} \frac{1}{2}\right)$ & -0.02 & -0.03 & 0.04 & 0.04 & -0.06 & -0.01 \\
\hline
\end{tabular}

mediated from the scattering of conduction electrons, giving rise to the saturated moment for $\mathrm{CeSb}$ and the magnetic moment collapse from CeSb to CeTe (see Table III).

Listed in Table III are the calculated zero-temperature moment and ordering temperature, $T_{N}$, from the second $a b$ initio based method, with and without the CF interaction. It is clear that for the heavier systems $(\mathrm{CeBi}, \mathrm{CeSb}, \mathrm{CeTe})$ the effect of the $\mathrm{CF}$ interaction on the magnetic moments is small and slightly more pronounced on the ordering temperatures. This is due to the fact that for the more localized systems the CF interaction is smaller than the exchange in-

TABLE III. Calculated (from the model Hamiltonian) values of the zerotemperature ordered moments $\left(\mu_{o}\right)$ and ordering temperatures, Néel temperature $\left(T_{N}\right)$ both without (no $\mathrm{CF}$ ) and with the crystal field splittig term $(\mathrm{CF})$, and the correspomding experimental values for the cerium compounds. The experimentsl values of the crystal field splitting $\left(\Delta_{\mathrm{CF}}\right)$ are also listed. All moments are given in units of $\mu_{B}$, the temperatures and the crystal field splitting are given in units of Kelvin.

\begin{tabular}{lrrrrrrrrr}
\hline \hline & & \multicolumn{3}{c}{$\mu_{o}$} & & \multicolumn{3}{c}{$T_{N}$} \\
\cline { 9 - 10 } \cline { 7 - 9 } & $\Delta_{\mathrm{CF}}$ & No CF & CF & $\exp$ & & No CF & CF & $\exp$ \\
\hline $\mathrm{CeS}$ & 140 & 1.80 & 0.73 & 0.57 & & 1.0 & 11.0 & 8.4 \\
$\mathrm{CeSe}$ & 116 & 1.10 & 0.79 & 0.57 & & 2.5 & 14.0 & 5.7 \\
$\mathrm{CeTe}$ & 32 & 0.60 & 0.46 & 0.30 & & 8.0 & 5.0 & 2.2 \\
$\mathrm{CeP}$ & 150 & 2.10 & 0.73 & 0.81 & & 14 & 11 & 8 \\
$\mathrm{CeAs}$ & 137 & 2.10 & 0.74 & 0.85 & & 16 & 13 & 8 \\
$\mathrm{CeSb}$ & 37 & 2.10 & 1.80 & 2.06 & & 20 & 18 & 17 \\
$\mathrm{CeBi}$ & 8 & 2.10 & 2.10 & 2.10 & & 40 & 40 & 26 \\
\hline \hline
\end{tabular}

teractions. This is the reason that previous calculations, neglecting the $\mathrm{CF}$ interaction, gave results in very good agreement with experiment. On the other hand, for the lighter more delocalized systems the $\mathrm{CF}$ interactions are much larger than the exchange interactions and hence dominate the magnetic behavior. The overall decrease of the magnetic moments in the presence of the CF interaction in all systems arises from the mixing of the off-diagonal angular momentum states $| \pm 5 / 2\rangle$ and $|\mp 3 / 2\rangle$ states from the CF interaction with $\Gamma_{7}$ ground state. Overall, we find an excellent agreement with experiment for both the zero-temperature moment and the ordered temperature (a more stringent test for the theory) using the second $a b$ initio based approach with all three pertinent interactions (hybridization, Coulomb exchange, and CF interactions) treated on an equal footing.

The research at California State University Northridge (CSUN) was supported by NSF under Grant No. DMR9531005, by the U.S. Army Grant No. DAAH04-95, and the CSUN Office of Research and Sponsored Projects, and at West Virginia University by the NSF under Grant No. DMR9120333.

${ }^{1}$ J. Rossat-Mignod, P. Burlet, S. Quezel, J. M. Effantin, D. Delacôte, H. Bartholin, O. Vogt, and D. Ravot, J. Magn. Magn. Mater. 31-34, 398 (1983).

${ }^{2}$ B. R. Cooper, R. Siemann, D. Yang, P. Thayamballi, and A. Banerjea, in The Handbook of the Physics and Chemistry of the Actinides edited by A. J. Freeman and G. H. Lander (North-Holland, Amsterdam, 1985), Vol. 2, Chap. 6, pp. 435-500.

${ }^{3}$ A. Dönni, A. Furrer, P. Fischer, and F. Hulliger, Physica B 186-188, 541 (1993)

${ }^{4}$ F. Hulliger, B. Natterer, and H. R. Ott, J. Magn. Magn. Mater. 8, 87 (1978).

${ }^{5}$ H. R. Ott, J. K. Kjems, and F. Hulliger, Phys. Rev. Lett. 42, 1378 (1979).

${ }^{6}$ J. Rossat-Mignod, J. M. Effantin, P. Burlet, T. Chattopadhyay, L. P. Regnault, H. Bartholin, C. Vettier, O. Vogt, D. Ravot, and J. C. Achart, J. Magn. Magn. Mater. 52, 111 (1985).

${ }^{7}$ N. Môri, Y. Okayama, H. Takahashi, Y. Haga, and T. Suzuki, Physica B 186-188, 444 (1993).

${ }^{8}$ R. J. Birgeneau, E. Bucher, J. P. Maita, L. Passell, and K. C. Turberfield, Phys. Rev. B 8, 5345 (1973).

${ }^{9}$ H. R. Ott, J. K. Kjems, and F. Hulliger, Phys. Rev. Lett. 42, 1378 (1979); F. Hulliger and H. R. Ott, J. Phys. C (Paris) 40, C5 (1979).

${ }^{10}$ J. Rossat-Mignod, J. M. Effantin, P. Burlet, T. Chattopadhyay, L. P. Regnault, H. Bartholin, C. Vettier, O. Vogt, D. Ravot, and J. C. Achart, J. Magn. Magn. Mater. 52, 111 (1985).

${ }^{11}$ D. L. Price and B. R. Cooper, Phys. Rev. B 39, 4945 (1989).

${ }^{12}$ O. Eriksson, M. S. S. Brooks, and B. Johansson, Phys. Rev. B 41, 7311 (1990).

${ }^{13}$ N. Kioussis (private communication).

${ }^{14}$ J. M. Wills and B. R. Cooper, Phys. Rev. B 36, 3809 (1987).

${ }^{15}$ N. Kioussis, B. R. Cooper, and J. M. Wills, Phys. Rev. B 44, 10003 (1991).

${ }^{16}$ Q. G. Sheng and B. R. Cooper, J. Appl. Phys. 69, 5472 (1991); Phys. Rev. B 50, 965 (1994).

${ }^{17}$ K. R. Lea, M. J. M. Leask, and W. P. Wolf, J. Phys. Chem. Solids 23, 1381 (1962). 